\title{
Serum Concentrations Of Lidocaine And Its Metabolites After Prolonged Infusion In Healthy Horses
}

\author{
Emma J. Dickey
}

Thesis submitted to the faculty of Biomedical and Veterinary Sciences in partial fulfillment of the requirements for the degree of

\author{
Master of Science \\ In \\ Biomedical and Veterinary Sciences
}

Harold C. McKenzie III (Chair)

Jennifer A. Brown

Martin O. Furr

Laura J. McCutcheon

June $11^{\text {th }} 2009$

Leesburg, Virginia

Keywords: Horse, Prokinetic, Glycinexylidide, Monoethylglycinexylidide, lleus 


\title{
Serum Concentrations of Lidocaine And Its Metabolites After Prolonged Infusion In Healthy Horses
}

By

\author{
Emma J. Dickey
}

\begin{abstract}
Lidocaine continuous-rate infusions $(\mathrm{CRI})$ are the most commonly used prokinetic in equine practice for the treatment of post-operative ileus and are also increasingly being used in pain management, such as in cases of severe laminitis, and are often used for prolonged durations. To date only limited time/concentration relationships of lidocaine administered as a short term (24hours) CRI to horses are reported. This study examined the time/concentration profile of lidocaine and its active metabolites, Monoethylglycinexylidide and glycinexylidide (MEGX, GX) during a 96 hour lidocaine infusion in eight mature healthy horses. Serum lidocaine concentrations reached steady state by three hours and did not accumulate thereafter. The serum concentration of lidocaine was above the target therapeutic concentration $(980 \mathrm{ng} / \mathrm{ml})$ only at 6 and 48 hours. The serum lidocaine concentration did not reach the range described as potentially causing toxicity $(>1850 \mathrm{ng} / \mathrm{ml})$. The MEGX metabolite did not accumulate over time, while the GX metabolite accumulated significantly up to 48 hours and then remained constant. The serum concentrations of lidocaine, MEGX and GX were below the limit of detection within 24 hours of discontinuation of the infusion. None of the horses developed any signs of lidocaine toxicity during the study. It was concluded that the
\end{abstract}


metabolism of lidocaine was not significantly impaired by prolonged infusion, contrasting with studies in dogs and humans. No adverse effects were observed in this study, which with the lack of lidocaine accumulation suggests that prolonged infusions are safe. However the accumulation of GX, a potentially toxic active metabolite, is cause for concern. 


\section{ACKNOWLEDGEMENTS}

I would like to acknowledge my committee members Dr Harold McKenzie, Dr Martin Furr, Dr Jennifer Brown and Dr Jill McCutcheon. I would also like to thank Elaine Meilahn, Kristin Tomsic and Tina Cooman for their help with the collection of data.

This study was funded by a grant from the Morris Animal Foundation. 


\section{ATTRIBUTION}

Several colleagues and coworkers aided in the writing and research behind several of the sections of this dissertation. A brief description of their background and their contributions are included here.

Dr Harold McKenzie III DVM MS DACVIM is the primary Advisor and Committee Chair. Dr McKenzie was key in the planning and execution of the study.

Furthermore, Dr McKenzie also provided invaluable assistance in the statistical analysis.

Dr Jennifer Brown DVM DACVS assisted in the planning and execution of the study and she used her extensive experience in the management of post operative colic patient to assist with the writing of the prokinetic section of the thesis.

Dr Martin Furr PhD DVM DACIM was integral in the review process of the thesis.

Dr Laura J McCutcheon PhD DVM DACVP was integral in the review process of the thesis. 


\section{TABLE OF CONTENTS}

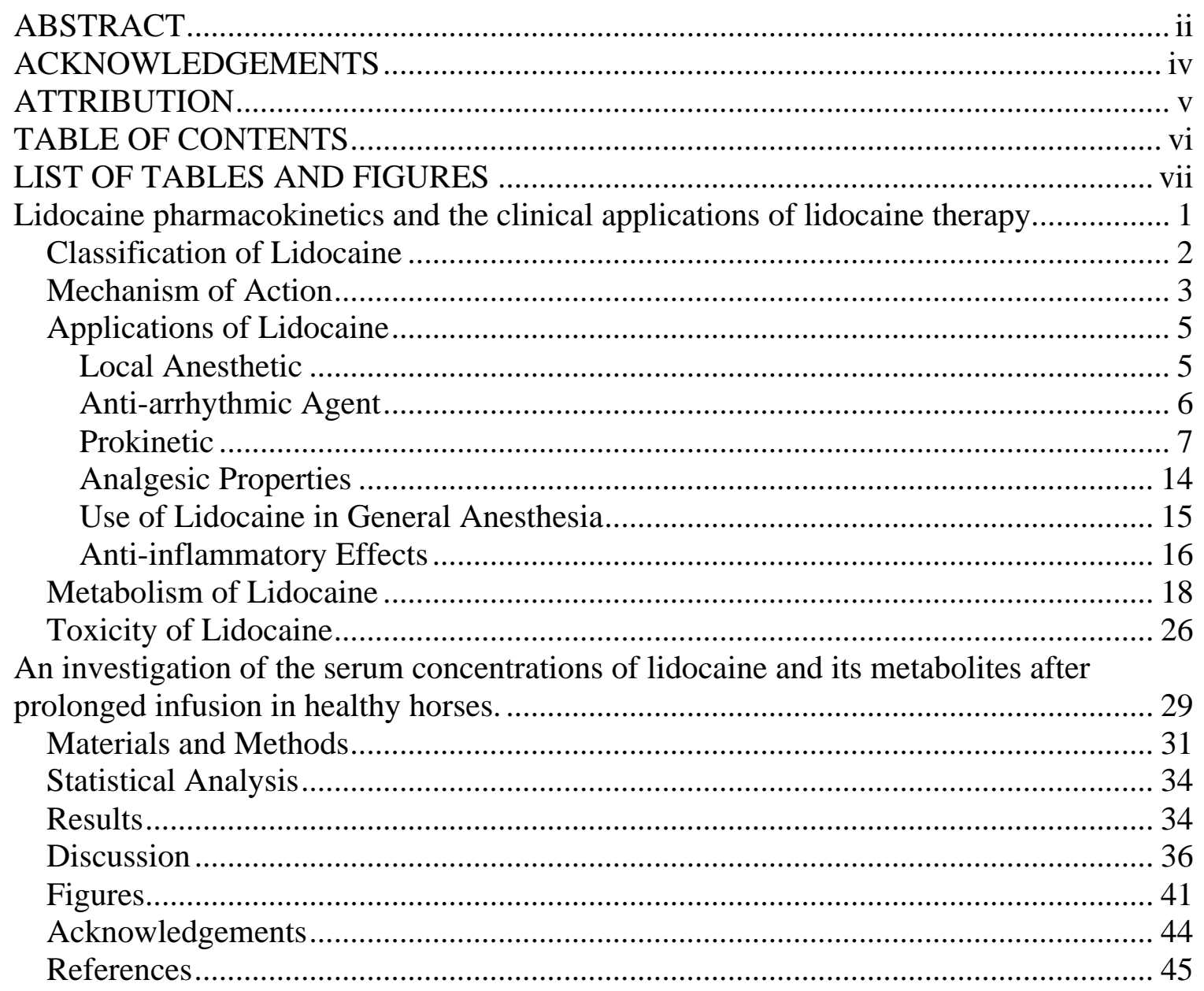




\section{LIST OF TABLES AND FIGURES}

Figure 1. Chemical structure of local anesthetics....................................

Figure 2. Mean concentrations of lidocaine and its metabolites GX and MEGX over time during lidocaine CRI........................................ 42

Figure 3. Mean concentrations of lidocaine and its metabolites GX and MEGX over time following discontinuation of lidocaine CRI........................43

Table 1. Clinical applications of lidocaine and the proposed mechanisms of action.......5

Table 2. Mechanisms of action and therapeutic effects of lidocaine................... 18

Table 3. Serum concentrations (mean, SEM) of lidocaine (L), MEGX and GX over the course of the 96 hour treatment interval..........................41 


\section{Lidocaine pharmacokinetics and the clinical applications of}

\section{lidocaine therapy.}

Lidocaine administered as a continuous rate infusion is now the most commonly used prokinetic therapy in equine medicine and has been shown to be effective in decreasing the duration of post operative reflux and in shortening the time to first passage of feces in horses following colic surgery. (Malone et al. 2006) In addition to their prokinetic effects intravenous infusions of lidocaine have proved to be effective in the management of chronic pain syndromes, including neuropathic pain in human patients. (Carroll et al. 2007; TremontLukats et al. 2006) Lidocaine is increasingly being used as a somatic analgesic in equine practice for the management of severe musculoskeletal pain, such as laminitis, and may have applications in ocular pain management. (Robertson 2004; Robertson et al. 2005) There is also a body of experimental evidence in rats and guinea pigs, which supports the use of lidocaine as an antioxidant and inflammatory modulator useful in preventing reperfusion injury. (Cassutto and Gfeller 2003)

In the clinical setting lidocaine is administered to equine cases as an initial bolus loading dose of $1.3 \mathrm{mg} / \mathrm{kg}$ bodyweight (over 15 minutes) followed by a continuous rate infusion of $0.05 \mathrm{mg} / \mathrm{kg} / \mathrm{min}$. (Malone et al. 2006) While initially proposed for use during the first 24 hours post surgery (Malone et al. 1998) the duration of the infusion is determined in clinical practice by the patient's individual needs and, as a result, the duration of infusion often exceeds 24 hours. Since 
previous studies have documented accumulation of lidocaine during and after prolonged intravenous infusions in human patients (LeLorier et al. 1977a; Milligan et al. 2006) this raises concerns about using prolonged infusions in horses. This concern is supported by a recent study which reported accumulation of lidocaine and it's metabolites glycinexylidide (GX) and monoethylglycinexylidide (MEGX) in horses receiving prolonged lidocaine continuous rate infusion following colic surgery. (de Solís and McKenzie 2007)

\section{Classification of Lidocaine}

Lidocaine is a local anesthetic widely used in both human and veterinary medicine. Local anesthetics are drugs which when applied to nerve tissue cause reversible blockage of nerve impulse conduction. Typically local anesthetics consist of an unsaturated aromatic group linked by an intermediate chain to a tertiary amine end, which itself acts as a proton acceptor. There are two main

groups of local anesthetics based on their intermediate chain. The aminoesters have an ester link between the aromatic and amine ends (Figure 1), examples include procaine and tetracaine. The aminoamides have an amide intermediate link and lidocaine is a member of this group. Other examples include mepivacaine, and bupivacaine. (Mama and Steffey 2001) 


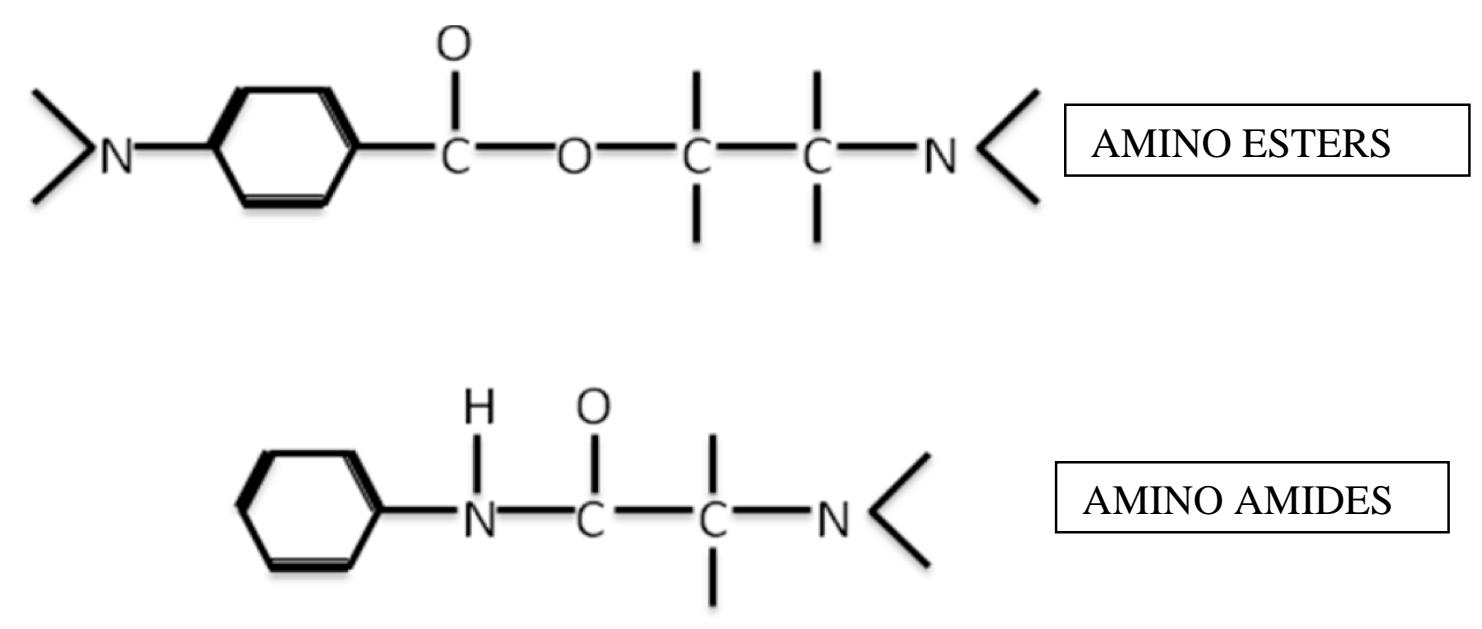

Figure 1. Chemical structure of local anesthetics

\section{Mechanism of Action}

Lidocaine inhibits the generation and conduction of nerve impulses by blockage of the voltage gated sodium channels in the nerve membrane. The resting membrane potential of large nerve fibers is $-90 \mathrm{mV}$, indicating that the inside of the nerve cell membrane is more negative than the outside of the fiber. This membrane potential is formed by the differential permeability of the nerve cell membrane to sodium and potassium ions. Of principal importance is the electrogenic sodium/potassium pump which expels three $\mathrm{Na}+$ ions to the outside for every two $\mathrm{K}+$ ions that are moved to the inside. This leaves a net deficit of positive ions and thus a negative potential inside the cell membrane. The 
partitioning of sodium and potassium across the nerve membrane causes a large concentration gradient across the nerve membrane. Another factor which determines the level of the normal resting membrane potential is the potassiumsodium leak channel, which normally is 100 times more permeable to potassium than sodium. Nerve signals are transmitted by rapid changes in membrane potential spreading along the length of the nerve fiber, otherwise known as an action potential. Depolarization occurs when there is a sudden increase in the permeability of the nerve fiber membrane to sodium, and due to the large concentration gradient sodium ions rapidly diffuse into the nerve cell causing the membrane potential to become more positive. Once a threshold potential is reached then an action potential is generated and nerve transmission can proceed. Shortly after the sodium channels open they begin to close and the potassium channels become more permeable than normal. This causes the rapid diffusion of potassium from the interior to the exterior to re-establish the normal resting membrane potential. This phase is known as repolarization.

The voltage gated sodium channel in the nerve membrane is the site of action of lidocaine and other local anesthetics. Due to the drug's lipophilic nature it diffuses into the lipoprotein membrane to bind to a receptor site in the sodium channel itself. This induces a conformational change in the channel and thus prevents the influx of $\mathrm{Na}+$ ions into the cell. By this means the action potential cannot propagate. Generally a higher frequency of stimulation and more positive membrane potential facilitate a greater degree of anesthetic block. This property 
is important in the use of lidocaine as a cardiac anti-arrhythmic drug as described later.

\section{Applications of Lidocaine}

\begin{tabular}{|c|c|}
\hline Clinical Application of Lidocaine & Proposed Mechanism of Action \\
\hline Local Anesthetic & Sodium channel blocker \\
\hline Anti-arrhythmic & Vaughn-Williams Class 1B \\
\hline Prokinetic & $\begin{array}{c}\text { Anti-inflammatory } \\
\text { Suppression of inhibitory reflexes }\end{array}$ \\
\hline Analgesic & $\begin{array}{l}\text { Sodium channel blocker } \\
\text { Possible NMDA antagonist }\end{array}$ \\
\hline Supplement to general anesthesia & $\begin{array}{c}\text { Reduces the MAC of inhalant from } \\
\text { analgesic properties reducing surgical } \\
\text { stimulation }\end{array}$ \\
\hline Anti-inflammatory & $\begin{array}{l}\text { Inhibition of granulocyte migration } \\
\text { Inhibition of free radical production }\end{array}$ \\
\hline
\end{tabular}

Table. 1. Clinical applications of lidocaine and the proposed mechanisms of action.

\section{Local Anesthetic}

In horses lidocaine $2 \%$ is a commonly used local anesthetic. It is used for perineural and epidural anesthesia, local skin block for laceration repair and local instillation into the airway to facilitate procedures such as bronchoalveolar lavage. Recently however $2 \%$ mepivacaine has become more popular for 
perineural anesthesia as it is considered to be less irritating and longer lasting than lidocaine. (Mama and Steffey 2001)

\section{Anti-arrhythmic Agent}

Lidocaine was first synthesized in 1946 and first used as an antiarrhythmic therapy in 1950. (Garfein 1982) In human patients it was extensively used in the 1970s-1990s as an intravenously administered medication for short term control of ventricular arrhythmias or for prophylactic treatment of arrhythmias in patients suffering from acute myocardial infarction. Lidocaine is the drug of choice for the treatment of ventricular tachycardia, as it is the most fast acting anti-arrhythmic drug available. According to the Vaughn Williams classification of anti-arrhythmics, lidocaine is a class IB drug.

Class I anti-arrhythmic agents block the voltage gated sodium channels of the myocardial cells. Importantly these drugs bind to sodium channels only when they are in the open or refractory states and dissociate from the channels in the closed state. This preferential binding of the lidocaine to activated sodium channels acts to block only the premature depolarization of damaged myocardial cells and therefore prevents re-entry pathways. (Muir and McGuirk 1985) The rapid binding and dissociation kinetics of these agents means that they are ineffective in controlling atrial arrhythmias since the atrial refractory period is very short. This class has no effect on potassium channels and therefore do not shorten the action potential or cause QT interval prolongation. Lidocaine acts mainly within the non-nodal tissue of the ventricle as a result of the longer ventricular refractory period. It is important to ensure that any electrolyte 
imbalances are first corrected as the drug is less effective in the presence of hypokalemia. In human patients lidocaine concentrations of $1.2-5 \mathrm{ug} / \mathrm{ml}$ will suppress ventricular ectopy in $80 \%$ of patients. (Pieper et al. 1982) In horses lidocaine has been successfully used in the treatment of ventricular arrhythmias and is widely used for this purpose. (Muir and McGuirk 1985) The recommended dose of lidocaine to treat ventricular tachycardia in horses is $0.25-0.5 \mathrm{mg} / \mathrm{kg}$ IV slowly to effect and this bolus can be repeated every 5-10 minutes to a total dose of $4 \mathrm{mg} / \mathrm{kg}$. (Muir and McGuirk 1985)

\section{Prokinetic}

Peristalsis of the small intestine is directly correlated to the migrating myoelectric complex (MMC). (Davies and Gerring 1983) The MMC is a wave of activity which passes through the intestinal tract in a regular cycle stimulating peristalsis. This is present in most species however the horse is unique in that there is no difference in the motility pattern between the fasted and fed states. (Koenig and Cote 2006) The MMC has three phases. Phase I is primarily slow waves which are subthreshold electrical potentials that do not cause smooth muscle contraction. Phase II has intermittently occurring spike bursts, and finally Phase III has spike bursts associated with each slow wave. (Milligan et al. 2007) Spike bursts are action potentials superimposed on the slow waves which result in contraction of the intestinal muscular layer. (Davies and Gerring 1983) The individual components of the $\mathrm{MMC}$, i.e. the duration of each phase and the spiking activity, can be used as a determinant of electrical activity. The spiking 
activity can then be used as an index of intestinal propulsive motility. (Scott et al. 2006)

Gastrointestinal ileus is defined as the functional inhibition of propulsive intestinal activity, irrespective of its pathophysiology. Post operative ileus is still a major problem in the management of colic, and is the leading cause of death in these patients. It has been found that the risk of developing post operative ileus is higher in cases with the following characteristics: $>10$ years old, Arabian breed, PCV $>45 \%$, increased total solids, anesthesia of $>2.5$ hours, surgery $>2$ hours, small intestinal lesions and anastomosis. (Roussel et al. 2001) Primary ileus is a major component of the syndrome proximal enteritis (or proximal duodenitisjejunitis, PDJ). Shock, electrolyte imbalance, hypoalbuminemia, peritonitis, endotoxemia, distention, ischemia, and inflammation of the intestinal tract have all been implicated in the etiology of ileus. (Dart and Hodgson 1998) Neural reflexes may mediate inhibition of motility associated with peritoneal inflammation. The afferent segment is composed partly of capsaicin-sensitive visceral afferent $\mathrm{C}$ fibers that terminate in the dorsal horn of the spinal cord where they can activate inhibitory sympathetic fibers or synapse directly on the sympathetic ganglia. Therefore the efferent limb of the reflex arc increases the sympathetic outflow and causes subsequent inhibition of acetylcholine release leading to ileus.

Several studies have shown an excitatory effect of local anesthetics on intestinal smooth muscle in vitro and in vivo, suggesting that these agents act by blocking inhibitory reflexes within the intestinal wall. (Rimback et al. 1990) One 
study investigated the treatment of postoperative paralytic ileus with intravenous lidocaine infusion in human patients. (Rimback et al. 1990) In this study thirty patients were admitted for cholecystectomy, and subsequently divided into two study groups. The treatment group received a $100 \mathrm{mg}$ lidocaine bolus prior to anesthesia, followed by a continuous rate infusion at $3 \mathrm{mg} / \mathrm{min}$. The infusion was continued for 24 hours after surgery. The placebo group received similar volumes of isotonic saline solution. The postoperative colonic motility was evaluated by radiopaque markers and serial abdominal radiographs. The authors found that there was a significantly earlier return of propulsive motility in the colon of the lidocaine treated patients with a mean colon transit time of 27 hours in the treated group versus 32 hours in the control group.

It has been shown previously that the amide class of local anesthetics has anti-inflammatory properties resulting from the reversible inhibition of both exocytosis of enzymes and generation of superoxide anion by polymorphonuclear cells. (Goldstein et al. 1977) Suggested underlying mechanisms include $\mathrm{Na}+/ \mathrm{Ca} 2+$ exchange inhibition, scavenging of superoxide and hydroxyl radicals and prevention of deleterious leukocyte-endothelial cell interactions. (Cassutto and Gfeller 2003) Results of several studies have suggested that nociceptive stimulation of the peritoneum from inflammation inhibits gastrointestinal motility by the activation of sympathetic inhibitory reflexes. (Kalff et al. 1998; Nadrowski 1983) Surgical manipulation of the intestine can result in the release of proinflammatory cytokines which initiate the inflammatory cascade and neutrophil chemotaxis into the intestine leading to 
post operative ileus. As outlined below evidence for lidocaine having a direct prokinetic effect is lacking and it is deemed likely that the action of lidocaine as a prokinetic is mediated through the reduction of inflammation. Rimback et al concluded that the reduction of postoperative peritoneal inflammation and subsequent suppression of inhibitory reflexes accounted for the earlier return of post operative colonic motility in the lidocaine treated patients. (Rimback et al. 1990)

One study investigated the in vitro effects of lidocaine on smooth muscle from the pyloric antrum, proximal duodenum and middle jejunum of horses. (Nieto et al. 2000) In this study isolated muscle strips from each portion of intestine were placed in an organ bath and attached to isometric force transducers. A selection of prokinetic agents including lidocaine was added in increasing concentrations and contractile activity subsequently recorded. The authors of that study found that lidocaine did not cause a significant increase in contractile amplitude of the pyloric antrum or mid jejunum. However there did appear to be an excitatory effect at the proximal duodenum shown by a significant dose dependent increase in contractile amplitude. (Nieto et al. 2000) One potential limitation of that study as noted by the authors is that the in vitro effects of lidocaine on smooth muscle isolated from clinically normal horses may not accurately reflect conditions in a clinical setting. Furthermore investigating the response in an isolated piece of tissue is obviously not representative of the in vivo situation. This is especially important as the proposed action of lidocaine is to inhibit neutrophil migration and cytokine release in the intestinal wall, so as 
soon as the intestinal tissue is removed from the body there is a lack of infiltrating polymorphonuclear cells. Also there is disruption of the neural reflex arc when using an isolated strip of tissue meaning that the lidocaine is unable to disrupt the inhibitory reflexes which are believed to result in ileus.

Milligan et al in 2007 investigated the effect of lidocaine on postoperative jejunal motility in normal horses. (Milligan et al. 2007) The horses in this study were anesthetized and electrical activity of the jejunum was recorded by suturing electrodes to the jejunum via a flank incision. The electrode signal was then converted to a myoelectric complex tracing. They reported that the duration of the MMC was unaffected by lidocaine administration as were the durations of Phases I and II. Although the duration of Phase III was shorter in response to lidocaine there was no change in the number of Phase III events or spiking activity. The authors concluded that the results did not support the use of lidocaine as a prokinetic. It is important however to note that again this study was performed in clinically normal horses and not those affected with post-operative ileus so the results may be somewhat expected based upon the proposed antiinflammatory mechanism of action of lidocaine.

Recently there have been some contradictory findings in the investigation of the efficacy of lidocaine as a prokinetic in the clinical setting. One study found that despite lidocaine treatment resulting in less jejunal distention and less abdominal fluid accumulation there was no significant difference in the presence of gastrointestinal sounds, time to passage of first feces, number of defecations in the first 24 hours, presence of gastric reflux, small intestinal contractions per 
minute assessed by ultrasonography, rate of complications or outcome. (Brianceau et al. 2002) Limitations of this study included the lack of gastric reflux in both the treatment and control groups which made it impossible to assess the effect of lidocaine on that particular variable. Furthermore a trend towards a reduction in the time to first defecation was noted in the treatment group, so possibly an increase in case number would have increased this to significance. Finally the authors made the observation that there was a low incidence of small intestinal lesions in this particular study again limiting the study of the effects of lidocaine in a group of cases at higher risk of developing post operative ileus.

An investigation by Malone et al in 2006 reported the use of intravenous continuous infusion of lidocaine for the treatment of equine ileus $(1.3 \mathrm{mg} / \mathrm{kg}$ bolus followed by an infusion of $0.05 \mathrm{mg} / \mathrm{kg} / \mathrm{min}$ ). (Malone et al. 2006) In contrast to the previously described study, these authors found that intravenous lidocaine administered after the start of reflux significantly decreased the amount of gastric reflux with $65 \%$ of lidocaine treated horses ceasing refluxing within 30 hours compared with only $27 \%$ of control horses. The lidocaine treated group also had earlier passage of feces and shortened hospital stay by a mean of $6+/-2.6$ days compared with placebo treated horses. The major difference between the two described studies is that the first study was looking at the pre-emptive use of lidocaine whereas Malone et al looked at the use of lidocaine once reflux had been observed. The findings of the second study underline the point made by Brianceau et al, that to further investigate the efficacy of lidocaine more cases at higher risk of post operative ileus needed to be included in the study group. 
A recent study by Rusiecki et al in 2008 investigated the effect of a lidocaine CRI on Gl function in normal horses. (Rusiecki et al. 2008) This study focused on the effect of lidocaine $(1.3 \mathrm{mg} / \mathrm{kg}$ bolus followed by $\mathrm{CRI}$ $0.05 \mathrm{mg} / \mathrm{kg} / \mathrm{min}$ ) on defecation frequency, weight of feces produced, intestinal transit time, fecal moisture content, and borborygmi. The subjects were monitored for any signs of lidocaine toxicity. The authors reported an increase in intestinal transit time and reduction in fecal output in the lidocaine treated horses but no effect on the fecal moisture content. Although this study was again performed in normal horses where there is no inflammation or pain induced inhibitory reflexes producing ileus it raises important questions as lidocaine CRI is increasingly being used as a somatic analgesic in otherwise healthy horses.

It is interesting to note that the in vitro studies and in vivo studies performed on normal horses have generally found that lidocaine does not have convincing prokinetic properties. On the other hand the studies that have found a positive effect have been performed in horses clinically affected by ileus. This can be explained by the current theory that lidocaine acts to reduce inflammation and pain and also disrupts the inhibitory neural reflexes associated with inflammation which are not present in clinically normal horses. 


\section{Analgesic Properties}

Sodium channel blockers are widely used for the treatment of neuropathic pain (Siddall and Cousins 1995) where they function by acting on nerve endings in the spinal cord and dorsal root ganglia. (Lauretti 2008) In chronic pain syndromes the dorsal horn neurons are highly sensitized, resulting in hyperalgesia and allodynia. It has been demonstrated that systemically administered lidocaine will preferentially act on these facilitated dorsal horn neurons due to higher frequency stimulation of the sodium channels leading to central analgesic effects (Sotgiu et al. 1992) Intravenously administered lidocaine reaches therapeutic concentrations within the cerebrospinal fluid where it inhibits muscarinic and glycine receptors and also acts as an NMDA antagonist which then modulates the release of excitatory neurotransmitters. (Lauretti 2008) At the peripheral level local anesthetics inhibit neuronal transduction, decrease the release of inflammatory mediators, inhibit neutrophil chemotaxis and reduce extravasation of albumin. (Erickson and Hultin 1992)

Lidocaine is increasingly being used in equine hospitals as an analgesic therapy, especially in the management of laminitis. (Malone and Graham 2002) A study performed in 2005 (Robertson et al. 2005) investigated the effect of systemic lidocaine on both visceral and somatic nociception in horses. To assess somatic analgesia thermal threshold was measured over the withers and it was found that lidocaine increased this threshold significantly at 30 and 90 minutes after the start of the infusion. To investigate the visceral analgesic properties colorectal and duodenal distention were measured. In this experiment there was 
no significant increase in the tolerance of visceral distention in response to lidocaine administration. From these findings it was concluded that while lidocaine may be very useful in the management of somatic pain it does not appear to be a good choice for visceral analgesia. This conclusion is also supported by a later study that found that lidocaine was an unsuitable choice as a sole analgesic in management of post operative colic cases, as horses treated with lidocaine alone showed an increase in pain score post operatively in comparison to flunixin meglumine. (Cook et al. 2008)

\section{Use of Lidocaine in General Anesthesia}

In addition to its potential role in analgesia lidocaine has been shown in humans, dogs, goats and horses to reduce the minimum alveolar concentration (MAC) of inhaled anesthetic agents (Doherty et al. 2007; Doherty and Frazier 1998; Himes et al. 1977) and therefore lidocaine infusions are widely used in standard anesthetic protocols. One study investigated the effect of intravenous lidocaine on halothane MAC in ponies. In that study six ponies were anesthetized and then maintained with halothane, and the MAC determined when administered lidocaine at 2.5 or $5 \mathrm{mg} / \mathrm{kg}$ bolus over 5 minutes followed by a continuous rate infusion of lidocaine (50 or $100 \mathrm{uk} / \mathrm{kg} / \mathrm{min}$ ) for 60 minutes. The authors found that a serum lidocaine concentration of $<2 \mathrm{ug} / \mathrm{ml}$ reduced the MAC of halothane by a maximum of $20 \%$, and serum concentration of $2.1-3.5 \mathrm{ug} / \mathrm{ml}$ decreased the MAC by $30-50 \%$. (Doherty and Frazier 1998) This MAC reducing effect is believed to be due to the central analgesic properties of lidocaine as a result of sodium channel blockade producing spinal and supraspinal analgesia.. 
One investigation reported the effects of intravenous lidocaine infusion on electroencephalogram (EEG) recordings during castration in ponies anesthetized with halothane. (Murrell et al. 2005) The authors reported that the administration of lidocaine prevented the shift towards higher frequency activity in the EEG which is usually seen in response to surgical stimulation, and concluded that this was consistent with the MAC halothane sparing effects of lidocaine. (Doherty and Frazier 1998; Murrell et al. 2005) Another study found that horses in which lidocaine infusion was continued until the end of surgery had a tendency toward a lower quality of recovery from anesthesia. (Valverde et al. 2005) Horses which received lidocaine until the end of surgery had a significantly higher ataxia score but no difference in the time to sternal recumbency, extubation or standing and no difference in the overall recovery score. Despite the overall recovery score not being affected by lidocaine the authors recommended that the lidocaine infusion should be discontinued thirty minutes prior to the end of surgery to avoid the risks of increased ataxia during the recovery period.

\section{Anti-inflammatory Effects}

There has been recent interest in the use of lidocaine to reduce reperfusion injury due to its ability to prevent reactive oxygen species formation and lipid peroxidation. It has been demonstrated that the amide local anesthetics have anti-inflammatory properties. (Goldstein et al. 1977) These antiinflammatory actions include the inhibition of prostaglandin synthesis, granulocyte migration, lysosomal enzyme release and free radical production. (Goldstein et al. 1977) Suggested mechanisms include $\mathrm{Na}+/ \mathrm{Ca}+$ exchange 
inhibition, scavenging of superoxide and hydroxyl radicals and prevention of deleterious leukocyte-endothelial cell interactions. (Cassutto and Gfeller 2003)

Myocardial reperfusion injury models have shown that inhibition of the $\mathrm{Na}+/ \mathrm{Ca} 2+$ exchange reduces the development of ventricular dysrhythmias and a reduction in the infarct size, by reduction of the accumulation of $\mathrm{Na}+$ and $\mathrm{Ca} 2+$ within the cardiac tissue. This mechanism is believed to be at work in neuronal tissue, with pre-ischemic administration of lidocaine to rats and gerbils inhibiting the release of intracellular $\mathrm{Ca} 2+$, and thus protecting against ischemic damage. (Liu et al. 1997) Further investigation showed that administration of intravenous lidocaine before reperfusion results in: reduced $\mathrm{Na}+$ and $\mathrm{Ca} 2+$ release from mitochondria (Terada et al. 1999), suppressed glutamate accumulation in hippocampal and cortical tissue, prevention of histological damage to hippocampal slices (Taylor et al. 1995) and reduction in infarct size, as well as improved neurological outcome and body weight. (Lei et al. 2001)

An investigation in 1992 found that lidocaine was a potent scavenger of hydroxyl radicals and singlet oxygen but not superoxide radicals. (Das and Misra 1992) Taniguchi et al found that administration of lidocaine immediately after the induction of endotoxemia inhibited the development of an inflammatory response, hypotension and metabolic acidosis in rabbits. (Taniguchi et al. 1996) A subsequent study performed by the same authors in 2000 found that the treatment with lidocaine immediately after intravenous administration of endotoxin resulted in a significant improvement in hemodynamics and inhibited the production of interleukin-6 and interleukin-8. 
The main clinical application of this feature of lidocaine is in the management and prevention of reperfusion injury in the equine gastrointestinal tract. It is widely accepted that ischemic lesions of the gastrointestinal tract have a poorer prognosis than simple obstructions. (Blikslager 2003) Most recently Cook et al (2008) investigated the ability of lidocaine to attenuate the deleterious effect of flunixin meglumine on the recovery of mucosal barrier function after ischemic injury. The authors found that lidocaine administration ameliorated the decrease in transepithelial resistance and increase in LPS permeability induced by flunixin meglumine. (Cook et al. 2008)

\begin{tabular}{|c|c|}
\hline Mechanism of action & Therapeutic effect \\
\hline Sodium channel blockade & Reduced sodium/calcium exchange \\
\hline Calcium channel blockade & Reduced xanthine oxidase activity \\
\hline Reduced glutamate release & Reduction of secondary brain injury \\
\hline Hydroxyl radical scavenging & Reduced lipid peroxidation \\
\hline Neutrophil respiratory burst inhibition & Reduced superoxide release \\
\hline Cytokine release inhibition & Reduced endothelial dysfunction \\
\hline
\end{tabular}

Table 2. Mechanisms of action and therapeutic effects of lidocaine. (Cassutto and Gfeller 2003)

\section{Metabolism of Lidocaine}

After intravenous administration the disposition of lidocaine in humans has been determined to be an open two compartment pharmacokinetic model with a 
fast distribution phase (half life 8.3minutes) and slower elimination phase (half life 107 minutes). (Sasyniuk and Ogilvie 1975) In cats however a three compartment model has been demonstrated. (Thomasy et al. 2005) As it takes approximately 5 half lives for lidocaine to reach steady state concentration this will require 9 hours in the normal patient (5 half lives x 107 minutes). (Waller 1981) For this reason in clinical cases an intravenous bolus is administered first to achieve therapeutic concentrations rapidly. Shortly after intravenous injection lidocaine is rapidly distributed to highly perfused tissues such as the brain and heart, such that within a short time period most of the drug has actually left the central blood pool. This occurs to such an extent that only $6 \%$ of the lidocaine in the body at steady state concentrations is within the blood pool. (Benowitz and Meister 1978) After this rapid distribution phase the lidocaine redistributes from the wellperfused tissues back to the blood pool and then to the muscle and adipose tissue. These tissues become the major storage reservoirs for the lidocaine, and later on when blood concentrations fall, the lidocaine is released back into the central circulation. (Benowitz and Meister 1978) Approximately $60 \%$ of the therapeutic concentration of lidocaine is plasma protein bound in the circulation. (Tucker et al. 1970)

Lidocaine is eliminated by hepatic metabolism and only about $3 \%$ of the dose is excreted as intact drug in the urine over 24 hours. Monoethylglycinexylidide (MEGX) is formed from lidocaine via oxidative $\mathrm{N}$ dealkylation by the hepatic cytochrome P450 system. MEGX which is further metabolized to glycinexylidide (GX) and 4-hydroxy-2,6-xylidine, is found only in 
small quantities in the urine (4\% of lidocaine dose). (Oellerich et al. 1987) Both major metabolites (MEGX and $\mathrm{GX}$ ) are considered to be pharmacologically active. MEGX is substantially active with both pharmacologic and toxicologic activity. (Smith and Duce 1971) GX however does not appear to have any antiarrhythmic effect but does contribute to central nervous system toxicity. (Blumer et al. 1973) The major hepatic metabolism system involved is thought to be the P450 isoenzyme CYP 3A4. (Oellerich and Armstrong 2001) It has been shown that $64 \%$ of lidocaine entering the liver is extracted during one passage through the liver. (Stenson et al. 1971) Due to this very high hepatic extraction ratio there is great dependency on hepatic blood flow for lidocaine metabolism, and factors that alter hepatic perfusion will therefore alter the rate of metabolism. (Garfein 1982) The half life of MEGX is reported to be 2.3 hours (Thomson et al. 1987) and that of GX has been calculated to be much longer at 9.8 hours. (Waller 1981)

There is a good correlation between lidocaine clearance and cardiac index (Thomson et al. 1973) and in normal patients clearance approaches hepatic blood flow. As a result of this property, MEGX formation kinetics have been used as an assessment of liver function. (Oellerich and Armstrong 2001) Patients with liver disease exhibit decreased clearance of lidocaine, which may be due to decreased hepatic blood flow, hepatocellular dysfunction or both. (Benowitz 1974) Additionally other drugs which affect hepatic microsomal enzyme systems either by inhibition or induction, such as cimetidine and chloramphenicol, may also influence lidocaine disposition. (Benowitz 1974) Lidocaine and MEGX 
concentrations are not altered in individuals with renal disease. $50 \%$ of $\mathrm{GX}$ is eliminated by renal excretion in humans, however, and this metabolite may accumulate in the presence of renal disease. (Mama and Steffey 2001)

The pharmacokinetics described above are only relevant for a single intravenous bolus administration of lidocaine, as the kinetics have been shown to change after prolonged infusions in human and canine patients, anesthetized patients and in disease states. The kinetics of lidocaine have been shown to be time dependent during prolonged intravenous infusions in human and canine patients. (LeLorier et al. 1977a; LeLorier et al. 1977b) Suggested causes for this include increases in alpha 1- acid glycoprotein levels, decreases in hepatic blood flow, saturation of metabolism, product inhibition and irreversible tissue binding to the liver (Tam et al. 1987) An increase in alpha 1-acid glycoprotein occurs in acute myocardial infarction, and this substance binds to lidocaine and decreases its clearance however this does not explain the time dependent kinetics in healthy subjects. (Bauer et al. 1982) An increase in volume of distribution could potentially result in a reduction in clearance and subsequent accumulation of lidocaine but no evidence of this has been found in healthy subjects. (Bauer et al. 1982) Product inhibition occurs when the presence of a metabolite of a drug actually inhibits further metabolism of the parent drug in a negative feedback type situation. This phenomenon has been investigated and it has been shown that MEGX and lidocaine compete for a hydroxylation pathway and therefore during a prolonged infusion the resultant increase the in the amount of MEGX causes a 
reduction in the hepatic metabolism of the parent drug lidocaine. (Thomson et al. 1987)

In a clinical setting lidocaine is often given as a bolus immediately followed by a continuous rate infusion. The duration of the infusion is determined by the patient's needs, and this often exceeds 24 hours. Experiments in man indicate that if the infusion lasts 12 hours or less then the disposition of the drug is similar to bolus administration. On the other hand if the infusion is prolonged the half life upon discontinuation of the drug averages 200 minutes. (LeLorier et al. 1977a) In that study lidocaine was administered as an intravenous bolus $(1 \mathrm{mg} / \mathrm{kg})$ followed by an infusion (20ug/ $/ \mathrm{kg} / \mathrm{min})$ lasting between 25 and 60 hours. The subjects had experienced uncomplicated myocardial infarction and the lidocaine was administered as a prophylactic anti-arrhythmic. The investigators found that the half life of lidocaine increased approximately two fold, and the clearance was decreased by approximately two-fold. This raises concerns about the accumulation of lidocaine or its metabolites to potentially toxic levels during a prolonged intravenous infusion.

A similar experiment was performed in dogs. (LeLorier et al. 1977b) This study looked at the elimination kinetics of a 90 minute infusion and a 24 hour infusion. These authors found that the elimination rate constant and clearance for lidocaine decreases by approximately one half during prolonged infusion. Due to the observation that both the elimination constant and the clearance decrease by the same factor this indicates that there is no apparent change in the volume of distribution with prolonged infusion. The hepatic extraction was $79.2 \%$ during the 
90 minute infusion but only $25.6 \%$ after 24 hours of infusion. This was explained by a decrease in the elimination capacity of the liver. A subsequent study performed in horses (Milligan et al. 2006) looked at the disposition of lidocaine during a 12 hour intravenous infusion to post operative horses. Plasma concentrations at time points $4-12$ hours $(2.45 \mathrm{ug} / \mathrm{ml})$ were significantly higher than those at $1-3$ hours $(1.5 \mathrm{ug} / \mathrm{ml})$, and the authors concluded that a reduction in infusion rate may be necessary after the first 3-4 hours of infusion to decrease the risk of toxicity. They also determined the degree of protein binding of lidocaine to be $53.06+/-10.28 \%$ which decreased to $27.33+/-9.72 \%$ and $29.52+/-6.44 \%$ when combined with ceftiofur and ceftiofur/flunixin combined respectively. They concluded that although the protein binding is moderate, highly protein bound drugs may displace lidocaine increasing the unbound concentrations and the risk of lidocaine toxicity.

A pilot study was performed at MDSEMC in 2004-2005 to investigate the concentrations of lidocaine and its two main metabolites MEGX and GX in clinical cases. (de Solís and McKenzie 2007) The subjects of the study were a group of 10 adult post operative colic horses that were receiving the standard prokinetic dose of lidocaine at $1.3 \mathrm{mg} / \mathrm{kg}$ IV over 15 minutes followed by a $\mathrm{CRI}$ at $0.05 \mathrm{mg} / \mathrm{kg} / \mathrm{min}$ for 48 hours or longer. This study found that therapeutic concentrations of lidocaine were reached between 3 and 6 hours of the CRI, and the mean concentration of lidocaine showed a progressive increase from initiation of therapy until 12 hours into the $\mathrm{CRI}$, and then increased moderately during the next 48 hours. Serum concentrations of lidocaine were lower in the 
group which received a $\mathrm{CRI}$ of less than 96 hours duration, as compared with those that received lidocaine for longer then 96 hours. Four horses in the study had lidocaine concentrations in the potentially toxic range $>1850 \mathrm{ng} / \mathrm{ml}$, however none of these showed adverse effects. The serum concentrations of MEGX and GX showed substantial inter-individual variability, however there was a steady increase in the mean values from baseline until 72 hours after initiation of the $\mathrm{CRI}$, and following this time point serum concentrations increased markedly until the end of the infusion. Again no adverse effects were noted when the serum concentration of either metabolite was at its highest. The concentrations of lidocaine and both metabolites decreased rapidly after discontinuation of therapy. The observations of high concentrations of metabolites and the lack of increased elimination half life both of the parent drug and the metabolites after discontinuation of the infusion suggest that the metabolism of lidocaine is not significantly impaired after a prolonged intravenous infusion in horses in a clinical setting. This suggested a different pattern than in other species after prolonged intravenous infusions. The high concentrations of MEGX and GX observed in that preliminary study and the progressively increasing concentrations of these potentially neurotoxic metabolites represented cause for concern.

In addition to the effects of duration of infusion a number of disease states have also been shown to change the disposition of lidocaine. In patients suffering from congestive heart failure there are two important findings, firstly the volume of distribution of the central compartment is smaller as a result of decreased perfusion. This reduction in Vd means that a smaller loading dose is required to 
achieve therapeutic plasma concentrations. Secondly in congestive heart failure there is a reduction in hepatic blood flow which directly leads to a reduction in lidocaine clearance. (Thomson et al. 1973; Waller 1981) Lidocaine disposition has also been investigated in human patients with renal disease. Since renal excretion accounts for such a small fraction of lidocaine elimination lidocaine clearance is unaltered by renal disease. However up to $50 \%$ of GX, which is a polar metabolite with toxic potential, is eliminated via the kidneys, and thus GX can accumulate in renal disease. (Thomson et al. 1973)

Humans suffering from hepatic dysfunction have reduced lidocaine clearance due to a reduction in microsomal enzyme activity. (Waller 1981) In recent years MEGX formation from lidocaine has been used as a dynamic test of hepatic function in the assessment of patients prior to and after receiving a liver transplant and also has value as a prognostic indicator. (Oellerich and Armstrong 2001) The test involves measuring MEGX concentrations before and 15 or 30 minutes after administration of $1 \mathrm{mg} / \mathrm{kg}$ lidocaine as an intravenous bolus. The authors found that patients with a MEGX 15 or 30 minute test value $<10 \mathrm{ug} / \mathrm{L}$ had a low 1 year survival rate. (Oellerich and Armstrong 2001)

The effects of general anesthesia on lidocaine metabolism have been investigated in humans, sheep, cats and horses. In all species studied higher plasma lidocaine concentrations were found at all time points, due to reduced lidocaine clearance associated with general anesthesia. (Feary et al. 2005; Mather et al. 1986; Thomasy et al. 2005) This change in disposition kinetics was 
explained by a reduction in cardiac index and subsequently a reduction in hepatic blood flow, similar to that described for congestive heart failure.

\section{Toxicity of Lidocaine}

In human subjects two main categories of lidocaine toxicity are described: central nervous system toxicity and hemodynamic effects. The most common manifestation of lidocaine toxicity in humans is neurological abnormalities, which can even be seen at therapeutic doses. Clinical signs include dizziness, drowsiness, and euphoria, and at higher doses patients may suffer from muscle fasciculations and seizures. At high lidocaine concentrations significant hemodynamic side effects can be seen which include impairment of myocardial contractility, peripheral vasodilation and subsequent hypotension. (Benowitz et al. 1974) Most often CNS disturbances are manifest before cardiovascular abnormalities. (Adriani et al. 1966) In one study lidocaine toxicity was observed in $6.3 \%$ of patients to whom it was administered and was considered life threatening in $1.6 \%$ of cases. (Pfeifer et al. 1976)

High plasma levels of lidocaine in the horse may result from inappropriate drug dosing or impaired elimination, and can cause toxicity affecting the central nervous system, cardiovascular and musculoskeletal systems. The signs of lidocaine toxicity reported in the horse include alteration in visual function, rapid eye blinking, anxiety, mild sedation, ataxia and collapse. (Brianceau et al. 2002; Malone et al. 2006; Meyer et al. 2001) Equine colic patients at the investigator's clinic (MDSEMC) that were receiving prolonged lidocaine infusions have been observed to develop ataxia, muscle tremors and altered mentation, raising 
concern that toxic levels may have been reached despite the use of the recommended dosage regimen. The target steady state concentration for the treatment of ileus in the horse is $980 \mathrm{ng} / \mathrm{ml}(1000-2000 \mathrm{ng} / \mathrm{ml}$ range), (Malone et al. 1998) and the serum lidocaine concentrations resulting in adverse side effects have been reported to be $>1850 \mathrm{ng} / \mathrm{ml}$. (Meyer et al. 2001) In that study the investigators did not note any cardiovascular anomalies, however behavioral abnormalities were observed at a serum concentration of $1.85-4.35 \mathrm{ug} / \mathrm{ml}$ (mean $3.24+/-740 \mathrm{ug} / \mathrm{ml})$. In human patients subjective side effects are reported at lidocaine concentrations above $3-5 \mathrm{ug} / \mathrm{ml}$ and objective toxicities observed above 6-10ug/ml. (Benowitz et al. 1974)

The toxic serum concentrations of MEGX or GX have not been determined in the horse, and the concentrations of lidocaine, MEGX and GX during prolonged intravenous infusion in healthy horses have not been described. The current recommended dose for lidocaine is an initial loading dose of $1.3 \mathrm{mg} / \mathrm{kg}$ (over 15 minutes) followed by a continuous rate infusion of $0.05 \mathrm{mg} / \mathrm{kg} / \mathrm{min}$ for awake horses after abdominal surgery. (Malone et al. 1998) The reported concentration of lidocaine during short term CRI in horses is very variable even when a similar dosage regimen is used, and in some cases the concentration exceeds the concentrations reported as toxic. (Brianceau et al. 2002; Doherty and Frazier 1998; Dzikiti et al. 2003; Feary et al. 2005; Robertson et al. 2005) Due to the lack of pharmacokinetic data of prolonged infusions in horses the following study was designed to report the serum concentrations of 
lidocaine and both its metabolites during and after discontinuation of a 96 hour infusion of lidocaine. 


\section{An investigation of the serum concentrations of lidocaine and its}

metabolites after prolonged infusion in healthy horses.

E. J. Dickey, H. C. McKenzie III, J. A. Brown and C. N. deSolis (Dickey et al. 2008)

This chapter was previously published in the Equine Veterinary Journal, Volume 40, pages 348-352 and is used with the kind permission of Peter D. Rossdale, Editor, EVJ, June 8, 2009. 


\section{Reasons for Performing Study}

Only limited time/concentration relationships of lidocaine administered as a short term (24hours) CRI to horses are reported in the literature, and no true assessment of the pharmacokinetics of $\mathrm{CRI}$ administration of lidocaine has been reported. Furthermore there is no information available regarding the pharmacokinetics of prolonged lidocaine $\mathrm{CRI}$ or of the kinetics of the metabolites MEGX and GX. A pilot study performed at the MDSEMC of 10 clinical cases receiving prolonged lidocaine infusions (3-5days) demonstrated only moderate lidocaine accumulation over time, while profound accumulations of $G X$ and MEGX were noted. No normal horses were examined and the study was not blinded or fully randomized, requiring further work in order to determine the causative factors underlying these findings. A more complete understanding of the kinetics of intravenous lidocaine therapy in horses may allow for modifications of the dosage regimen resulting in improved patient safety, and will provide a more concrete basis for determining the appropriateness of prolonged lidocaine $\mathrm{CRI}$ therapy.

The first objective of this study was to determine the time/concentration profile of lidocaine administered as a prolonged intravenous infusion (96 hours) to healthy horses. The second objective was to determine if accumulation of lidocaine or its active metabolites MEGX and GX occurred during prolonged intravenous infusion. Our hypothesis was that accumulation of lidocaine and/or its active metabolites MEGX and GX would occur during prolonged continuous intravenous infusion of lidocaine in healthy horses. 


\section{Materials and Methods}

Eight mature (mean 9 years, range: $4-11$ years old) Standardbred horses were used for the study. There were 5 mares and 3 geldings in the study group. All horses were clinically healthy based on clinical examination, normal CBC and serum biochemistry profile, including hepatic parameters. After purchase the horses were dewormed with an ivermectin and praziquantel combination product'. Horses were maintained at pasture when not actively involved in the study. When participating in the study the horses were housed in 12 foot by 12 foot box stalls and had free access to water and hay during the experiment. During the study twice daily clinical examinations, including complete neurological evaluation, were performed by one of the investigators.

The determination of eight subjects was derived by the power analysis of the pilot study. This determination was based upon the base mean half life of 48 minutes for lidocaine and a population standard deviation of 5 , given that normal healthy horses were being used. With alpha at 0.05 at least 4 horses would be required in each group to detect a difference in the half life between the groups. This study was approved by the Virginia Polytechnic Institute and State University Animal Care and Use Committee (VPIACUC).

A 2 by 2 latin square crossover study design was used. The 8 horses were randomly divided into 2 groups of 4 horses each, with one group receiving $\mathrm{CRI}$ lidocaine in the first phase of the study followed by bolus lidocaine in the second phase, while the other group received bolus lidocaine in the first phase and CRI 
lidocaine in the second phase. One phase of the crossover consisted of the administration of a single intravenous bolus of lidocaine $(1.3 \mathrm{mg} / \mathrm{kg})$ administered over 15 minutes, with serum samples subsequently collected for lidocaine, MEGX and GX determination over a 24 hour period. Samples were taken at the following times (T hours); T0 (prior to lidocaine administration), T1, T2, T3, T6, T12, T18 and T24. The other phase of the crossover consisted of administration of a lidocaine CRI $(0.05 \mathrm{mg} / \mathrm{kg} / \mathrm{min})$ over a 96 -hour period. No bolus was administered prior to initiation of the CRI. Samples were collected at times (Thours); T0 (prior to CRI), T3, T6, T12. T24, T48, T72, T96. Samples were also collected after the discontinuation of the lidocaine CRI (TD hours); TD1, TD6, TD12, TD24. There was a two week washout period between each phase of the crossover.

Lidocaine was prepared as a $3.3 \mathrm{mg} / \mathrm{ml}$ solution by placing 10 grams of lidocaine solution" into 3 litres of Lactated Ringers Solutioniii. The CRI was controlled by use of an intravenous fluid administration pump ${ }^{\mathrm{iv}}$. The lidocaine solution was administered via sterile infusion tubing which terminated in coiled extension tubing ${ }^{v}$, which allowed the horse free movement within the box stall at all times. A single 14 gauge, $13 \mathrm{~cm}$ long polyurethane indwelling intravenous catheter ${ }^{\mathrm{vi}}$ was aseptically placed into both the left and right jugular veins of the horses prior to commencement of each phase of the study. The skin at the area of catheter placement was anesthetized with $1 \mathrm{ml}$ of mepivacaine ${ }^{\mathrm{vii}}$ prior to sterile preparation of the site and sterile catheter placement. The catheter in the right jugular vein was used for collection of blood samples, and the catheter in the left 
jugular vein was used for the administration of lidocaine. For sample collection an initial $10 \mathrm{ml}$ of blood was discarded and then the $10 \mathrm{ml}$ blood sample was placed into non anti-coagulated vacuum tubes ${ }^{\text {viii. }}$. This was carried out by the principal investigator, co-investigators or designated veterinary technicians. Following sample collection the catheter was flushed with $10 \mathrm{ml}$ of heparinised $0.9 \%$ sodium chloride, and the catheter was also flushed at 6 hour intervals throughout the study period. After collection the blood samples were allowed to clot then separated within 30 minutes of collection by centrifugation at 3000 rpm for 10 minutes. One millilitre aliquots of serum were then frozen at $-80 \mathrm{C}$ until assayed.

To determine the serum concentration of lidocaine, MEGX and GX a previously validated high performance liquid chromatography assay was used (Doherty and Frazier 1998). This analysis was performed by an external laboratory ${ }^{\mathrm{ix}}$. The frozen serum samples were submitted to the laboratory on dry ice. The HPLC procedure is based upon well described techniques (Dusci and Hackett 1985; Weiker et al. 1991) which were adapted and validated by Doherty et al (Doherty and Frazier 1998) in the assessment of the effect of lidocaine on the MAC of halothane in anesthetized ponies. The same technique was used in a recent clinical study of prolonged lidocaine CRI in clinically ill horses (de Solís and McKenzie 2007). After thawing the serum samples were vortexed and the lidocaine and metabolites extracted into dichloromethane $(4 \mathrm{ml})$ from alkalinized serum $(200 \mu \mathrm{l}$ sodium hydroxide, $1 \mathrm{~mol} / \mathrm{l})$. The organic layer was evaporated under nitrogen and the dried residue was reconstituted with $1 \mathrm{ml}$ of mobile phase (isocratic mixture of $0.03 \mathrm{~mol} / /$ potassium dihydrogen phosphate:acetonitrile 
$(87: 13 \mathrm{v} / \mathrm{v})) .180 \mu \mathrm{l}$ of the reconstituted sample was injected onto an $8 \times 100 \mathrm{~mm}$ $\mu$ Bondapak C18 cartridge ${ }^{x}$. The analytical system consisted of a Model 600 solvent delivery system, a Model 712 WISP auto sampler, a Model 481 absorbance detector, and a computer equipped with 820 Maxima software ${ }^{\mathrm{x}}$. Column effluent was monitored using ultraviolet detection at $205 \mathrm{~nm}$. Lidocaine, MEGX and $\mathrm{GX}$ concentrations were reported as $\mathrm{ng} / \mathrm{ml}$ serum.

\section{Statistical Analysis}

Lidocaine, MEGX and GX concentration data were first analyzed using basic descriptive statistics to calculate the mean and the standard error of the mean (SEM) for all data sets. Values for lidocaine, MEGX and GX were evaluated for normality using the Shapiro-Wilk method with $\alpha=0.05$. Normally distributed data were analyzed by use of repeated measures ANOVA to detect any significant differences in the concentrations of lidocaine, MEGX and GX over

time. Predetermined post hoc comparisons were made by use of a least square mean with a Bonferroni correction. A value of $p<0.05$ was considered significant for all tests.

\section{Results}

None of the horses developed any clinical signs of lidocaine toxicity during the study. In phase 1 of the study the lidocaine, MEGX and GX concentrations were below the lower limit of detection of $50 \mathrm{ng} / \mathrm{ml}$ at all time points. The mean 
concentrations ( \pm SEM) of lidocaine, MEGX and $G X$ at each time point in phase 2 are displayed in Table 1. Serum concentration of lidocaine was above the target therapeutic level $(980 \mathrm{ng} / \mathrm{ml})$ only at 6 and 48 hours of infusion (figure 1$)$. All other time points were slightly below this target level. No significant increase in the concentration of lidocaine was observed from 3-96 hours. Lidocaine concentration reached steady state by 3 hours of infusion, with a mean lidocaine concentration of $938.5( \pm 25.2) \mathrm{ng} / \mathrm{ml}$ from 3 to 96 hours. The serum concentration of lidocaine did not reach the range described as potentially causing toxicity $(>1850 \mathrm{ng} / \mathrm{ml})$ at any time point.

The serum MEGX concentration reached an apparent steady state concentration of $\sim 450 \mathrm{ng} / \mathrm{ml}$ by the six hour time point of the $\mathrm{CRI}$, however there was no significant increase in the concentration of MEGX after the 3 hour time point. There was a statistically significant increase $(p=0.004)$ in $G X$ concentration over time from 3 hours to 48 hours, with the mean concentration rising from $368.50 \mathrm{ng} / \mathrm{ml}$ at 3 hours to $1226.88 \mathrm{ng} / \mathrm{ml}$ at 48 hours. There was no significant increase in the concentration of GX from 48 hours onward, however, and the average concentration of GX observed from 48 to 96 hours was $\sim 1170 \mathrm{ng} / \mathrm{ml}$. This accumulation of GX over time is illustrated in Figure 1. The 48 and 96 hour concentrations were significantly different from the 3 hour concentration $(p=0.015$ and 0.016 respectively), while the 72 hour concentration was not $(p=0.13)$.

Concentrations of lidocaine, MEGX and GX after discontinuation of the infusion are shown in Figure 2. The serum concentrations of all three compounds decreased quickly, and none were detectable 24 hours after discontinuation of 
the lidocaine. Pharmacokinetic analysis following discontinuation of the infusion was attempted, however due to the rapid elimination of the parent drug and its metabolites there were inadequate data points available for analysis.

\section{Discussion}

In the clinical setting lidocaine is administered as a bolus immediately followed by a continuous rate infusion. The duration of the infusion is determined by the patient's needs, and this often results in periods of administration which exceed 24 hours. As previous studies have documented systemic accumulation of lidocaine during and after prolonged intravenous infusions, this raises concerns about using prolonged infusions in horses. This study found that the lidocaine serum concentrations reached steady state by 3 hours, did not accumulate over time and decreased rapidly to undetectable levels after the infusion was discontinued. Similarly the MEGX metabolite did not accumulate over the course of the 96 hour lidocaine infusion, having reached a steady state by the six hour time point. There was, however, a dramatic increase in the serum concentration of GX from 3-48 hours, ultimately reaching concentrations $>1200 \mathrm{ng} / \mathrm{ml}$. After 48 hours the serum concentration of GX appeared to plateau, and the concentration decreased to undetectable levels within 24 hours of discontinuation of the infusion.

A bolus of lidocaine was not administered prior to initiation of the $\mathrm{CRI}$ in this experimental protocol, which differs from the regimen used in clinical cases. The goal of the study was to examine the time/concentration profile of a lidocaine 
$\mathrm{CRI}$ without the potential interference from a bolus, and given the 96-hour duration of the study any influence of the bolus would have been undetectable. It was not the aim of this investigation to define the pharmacokinetic profile of a bolus of lidocaine, but the crossover did utilize a bolus dose in the interest of comparing the elimination characteristics of lidocaine after short term infusion to that observed with long term infusion. The results of the bolus phase of the crossover were unexpected in that while lidocaine, MEGX and GX were detected in horses after the bolus, the mean concentrations were always below the lower limit of detection (LLD) of $50 \mathrm{ng} / \mathrm{ml}$ (data not shown). From this data it is clear that any influence the bolus on the CRI would have been very transient. Earlier and more frequent sampling after the bolus would have been allowed for more accurate assessment, however this did not affect the CRI phase of the study.

Interestingly the lidocaine concentration during the infusion was above the therapeutic threshold of $980 \mathrm{ng} / \mathrm{ml}$ at only 6 and 48 hours, and the mean steady state concentration was only $938.5 \mathrm{ng} / \mathrm{ml}$ from 3 to 96 hours. This is similar to the findings by Navas de Solis et al., (de Solís and McKenzie 2007) and was unexpected given that several studies have reported on this dosage regime in horses. In the previous clinical study there were concerns that intermittent discontinuation of the lidocaine infusion might have accounted for this finding, but that was not an issue with the current study. There were only two episodes of brief interruption (15 minutes or less) in the lidocaine infusion due to technical issues. In only one instance did this occur in temporal proximity to a sample collection, and this sample was not included in the analysis. Conversely, serum 
lidocaine concentrations did not at any time point reach concentrations in the range described as potentially toxic (>1850ng/ml) (Brianceau et al. 2002; Malone et al. 2006; Meyer et al. 2001).

After the infusion was discontinued the serum lidocaine concentration rapidly decreased to undetectable levels. The lack of lidocaine accumulation during the infusion and the rapid elimination of lidocaine after the infusion are interesting in that these findings differ from previous studies in dogs and humans, where long term intravenous infusions of lidocaine have been shown to cause a significant prolongation of the serum half life (LeLorier et al. 1977a; LeLorier et al. 1977b). Experiments in man indicate that if the infusion lasts 12 hours or less then the disposition of the drug is similar to bolus administration. In prolonged infusions the elimination half life of lidocaine has been demonstrated to increase approximately two-fold from 100 to 200 minutes (LeLorier et al. 1977a). A similar experiment was performed in dogs (LeLorier et al. 1977b). This study looked at the elimination kinetics of a 90 minute infusion and a 24 hour infusion. The findings were similar to those of previous studies in that the disposition of lidocaine was slower after an infusion lasting 24 hours or more, than after a bolus or short term infusion. A subsequent study performed in horses (Milligan et al. 2006) looked at the disposition of lidocaine during a 12 hours intravenous infusion to post operative horses. The authors found that the plasma concentrations at time points $4-12$ hours were significantly higher than those at 1 3 hours, and concluded that a reduction in infusion rate may be necessary after the first 3-4 hours of infusion to decrease the risk of toxicity. The results of the 
present study and that of de Solis and McKenzie suggest that reduction in dosage could lead to sub-therapeutic concentrations of lidocaine, but further studies in clinically ill horses are required.

In humans administered prolonged lidocaine CRI not only does the lidocaine concentration tend to increase over time, but the concentrations of the metabolites remain stable or even decrease. This has been attributed to a decrease in hepatic extraction ratio. In this study there was dramatic accumulation of GX during the infusion, although the level returned to baseline within 24 hours after discontinuation of the infusion. This differs from the findings of the previous study in post-operative colic patients, which reported an accumulation of both GX and MEGX over time. A potential explanation for this is that the previous study examined clinically-ill horses. Hemodynamic alterations are not uncommon in post-operative colics, and any change in the hepatic blood flow could have inhibited lidocaine metabolism, thereby inhibiting the production of MEGX and GX. Alternatively the presence of systemic inflammation may have altered lidocaine metabolism and the elimination of MEGX and GX, similar to the findings reported in a murine model of endotoxemia. (McKindley et al. 2002) Another point to consider is that GX is a product of MEGX metabolism so in the pilot study the hepatic metabolism of MEGX to GX may have been slower than that in the healthy horses in the current study therefore resulting in accumulation of MEGX and lessening the accumulation of GX. It is interesting that in both of these studies it is the metabolite rather than the parent drug which accumulates. Although the toxic levels of neither GX nor MEGX have been determined in the 
horse, the potential risk of these accumulations cannot be ignored. While none of the horses in this study showed any adverse effects, further investigations into the effects of these metabolites and their potential toxic concentrations are required. 


\section{Figures}

$\begin{array}{cccc}\text { Time (hrs) } & \text { Mean L (ng/ml) } & \text { Mean MEGX (ng/ml) } & \text { Mean GX (ng/ml) } \\ 0 & 0.0(0.0) & 0.0(0.0) & 0.0(0.0) \\ 3 & 947.25(54.60) & 322.38(50.80) & 368.50(59.50) \\ 6 & 1019.50(51.20) & 448.25(50.27) & 604.38(108.10) \\ 12 & 940.88(54.70) & 421.50(65.45) & 770.75(128.20) \\ 24 & 902.88(64.50) & 452.50(56.04) & 946.50(178.70) \\ 48 & 997.50(56.80) & 471.63(63.16) & 1226.88(198.70)^{a} \\ 72 & 950.13(60.60) & 422.88(48.60) & 1055.13(222.50) \\ 96 & 946.00(47.40) & 529.38(63.06) & 1234.13(220.60)^{a}\end{array}$

Table 3. Serum concentrations (mean, SEM) of lidocaine (L), MEGX and GX over the course of the 96 hour treatment interval. Significant differences from the 3 hour concentration are denoted by the symbol ${ }^{\mathrm{a}}$. 


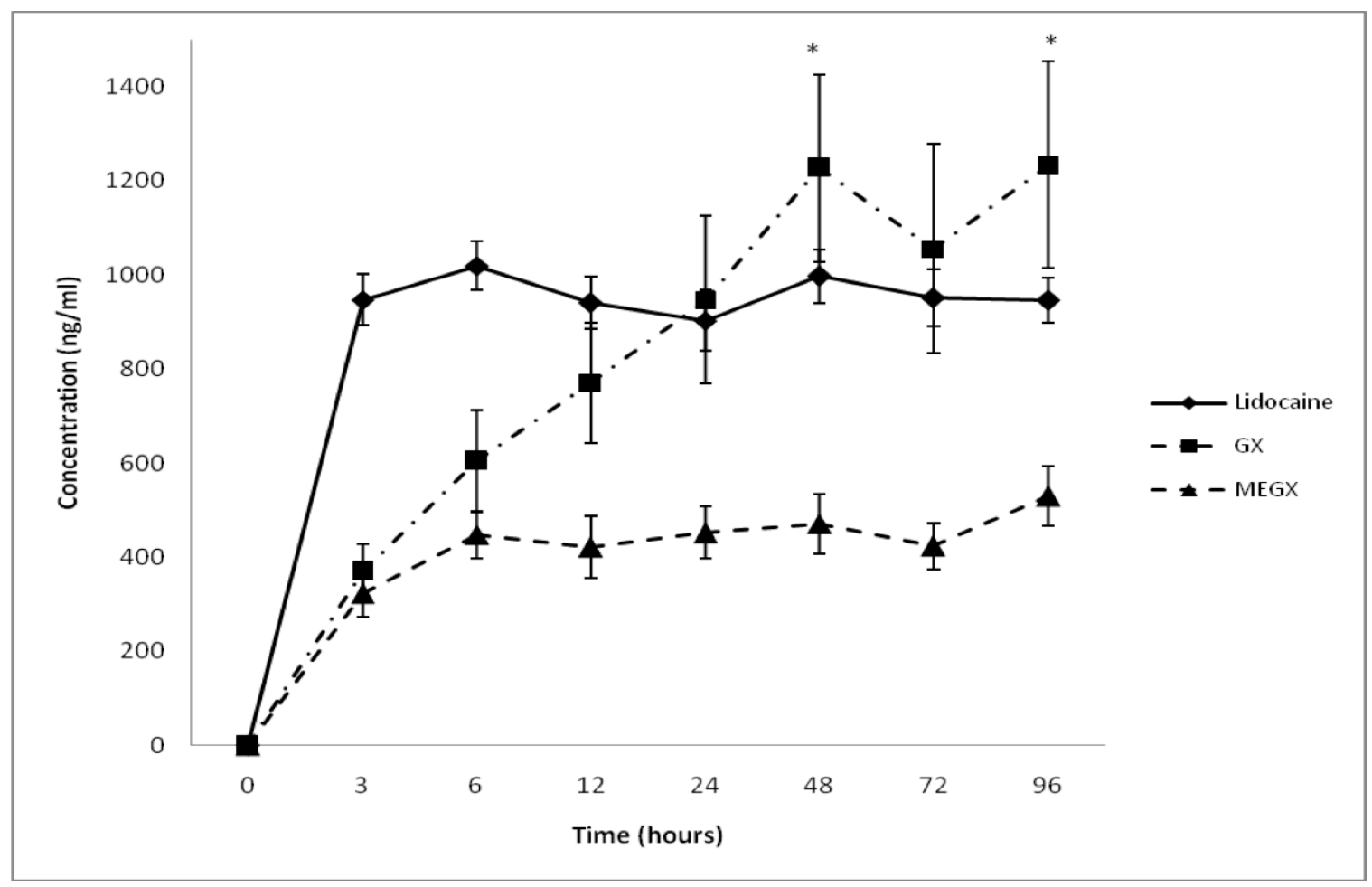

Figure 2. Mean concentrations of lidocaine and its metabolites GX and MEGX over time during lidocaine CRI. The error bars represent the standard error of the mean (SEM). * denotes significant difference from the 3 hour concentration. 


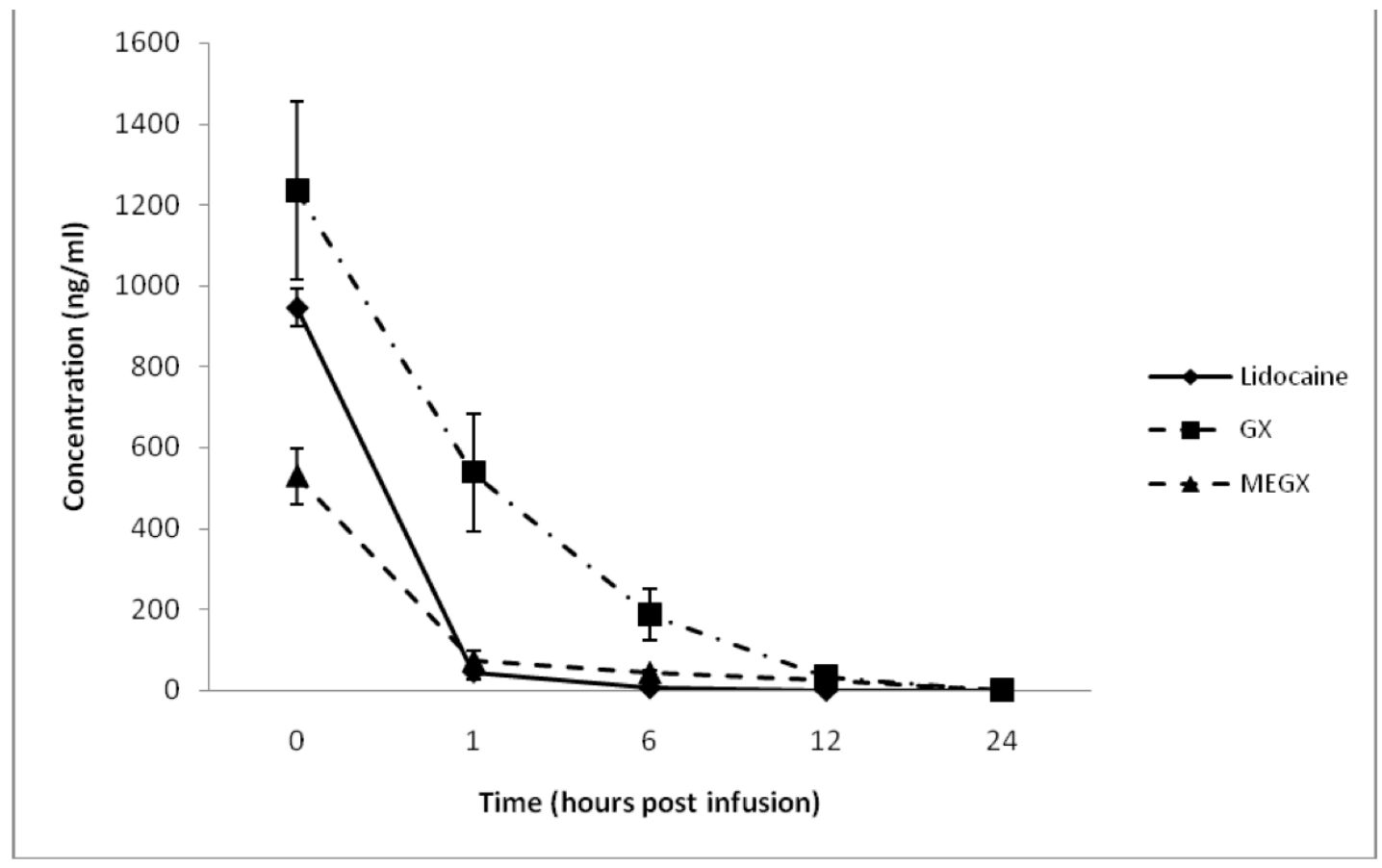

Figure 3. Mean concentrations of lidocaine and its metabolites GX and MEGX over time following discontinuation of lidocaine CRI. The error bars represent the standard error of the mean (SEM). 


\section{Acknowledgements}

This study was funded by a grant from the Morris Animal Foundation. The authors wish to thank Elaine Meilahn, Kristin Tomsic and Tina Cooman for their assistance with the animal phase of the study, and Dr. Sherry Wilcox of the

University of Tennessee, College of Veterinary Medicine, Department of Comparative Medicine, for performing the lidocaine and metabolite assays. 


\section{References}

Adriani, J., Zepernick, R. and Hyde, E. (1966) Influence of the status of the patient on systemic effects of local anesthetic agents. Anesth Analg 45, 87-92.

Bauer, L.A., Brown, T., Gibaldi, M., Hudson, L., Nelson, S., Raisys, V. and Shea, J.P. (1982) Influence of long-term infusions on lidocaine kinetics. Clin Pharmacol Ther 31, 433-437.

Benowitz, N., Forsyth, F.P., Melmon, K.L. and Rowland, M. (1974) Lidocaine disposition kinetics in monkey and man. I. Prediction by a perfusion model. Clin Pharmacol Ther 16, 87-98.

Benowitz, N.L. (1974) Clinical applications of the pharmacokinetics of lidocaine. Cardiovasc Clin 6, 77-101.

Benowitz, N.L. and Meister, W. (1978) Clinical pharmacokinetics of lignocaine. Clin Pharmacokinet 3, 177-201.

Blikslager, A.T. (2003) Treatment of gastrointestinal ischemic injury. Vet Clin North Am Equine Pract 19, 715-727.

Blumer, J., Strong, J.M. and Atkinson, A.J., Jr. (1973) The convulsant potency of lidocaine and its N-dealkylated metabolites. J Pharmacol Exp Ther 186, 31-36.

Brianceau, P., Chevalier, H., Karas, A., Court, M.H., Bassage, L., Kirker-Head, C., Provost, P. and Paradis, M.R. (2002) Intravenous lidocaine and small-intestinal size, abdominal fluid, and outcome after colic surgery in horses. $J$ Vet Intern Med 16, 736-741.

Carroll, I., Gaeta, R. and Mackey, S. (2007) Multivariate analysis of chronic pain patients undergoing lidocaine infusions: increasing pain severity and advancing age predict likelihood of clinically meaningful analgesia. Clin J Pain 23, 702-706.

Cassutto, B. and Gfeller, R. (2003) Use of intravenous lidocaine to prevent reperfusion injury and subsequent multiple organ dysfunction syndrome. Journal of veterinary emergency and critical care 13, 137-148.

Cook, V.L., Jones Shults, J., McDowell, M., Campbell, N.B., Davis, J.L. and Blikslager, A.T. (2008) Attenuation of ischaemic injury in the equine jejunum by administration of systemic lidocaine. Equine Vet J 40, 353-357. 
Dart, A.J. and Hodgson, D.R. (1998) Role of prokinetic drugs for treatment of postoperative ileus in the horse. Aust Vet J 76, 25-31.

Das, K.C. and Misra, H.P. (1992) Lidocaine: a hydroxyl radical scavenger and singlet oxygen quencher. Mol Cell Biochem 115, 179-185.

Davies, J.V. and Gerring, E.L. (1983) Electromechanical activity of the equine small intestine and its correlation with transit of fluid through Thiry-Vella loops. Res Vet Sci 34, 327-333.

de Solís, C.N. and McKenzie, H.C., III (2007) Serum concentrations of lidocaine and its metabolites MEGX and GX during and after prolonged intravenous infusion of lidocaine in horses after colic surgery. Journal of Equine Veterinary Science 27, $398-404$.

Dickey, E.J., McKenzie, H.C., 3rd, Brown, K.A. and de Solis, C.N. (2008) Serum concentrations of lidocaine and its metabolites after prolonged infusion in healthy horses. Equine Vet J 40, 348-352.

Doherty, T., Redua, M.A., Queiroz-Castro, P., Egger, C., Cox, S.K. and Rohrbach, B.W. (2007) Effect of intravenous lidocaine and ketamine on the minimum alveolar concentration of isoflurane in goats. Vet Anaesth Analg 34, 125-131.

Doherty, T.J. and Frazier, D.L. (1998) Effect of intravenous lidocaine on halothane minimum alveolar concentration in ponies. Equine Vet J 30, 300-303.

Dusci, L.J. and Hackett, L.P. (1985) Simultaneous determination of lidocaine, mexiletine, disopyramide, and quinidine in plasma by high performance liquid chromatography. J Anal Toxicol 9, 67-70.

Dzikiti, T.B., Hellebrekers, L.J. and van Dijk, P. (2003) Effects of intravenous lidocaine on isoflurane concentration, physiological parameters, metabolic parameters and stress-related hormones in horses undergoing surgery. J Vet Med A Physiol Pathol Clin Med 50, 190-195.

Erickson, M.C. and Hultin, H.O. (1992) Influence of histidine on lipid peroxidation in sarcoplasmic reticulum. Arch Biochem Biophys 292, 427-432.

Feary, D.J., Mama, K.R., Wagner, A.E. and Thomasy, S. (2005) Influence of general anesthesia on pharmacokinetics of intravenous lidocaine infusion in horses. Am J Vet Res 66, 574-580.

Garfein, O.B. (1982) Pharmacology of commonly used antiarrhythmic drugs and comments on the use of therapeutic drug monitoring. Ther Drug Monit 4, 1-14. 
Goldstein, I.M., Lind, S., Hoffstein, S. and Weissmann, G. (1977) Influence of local anesthetics upon human polymorphonuclear leukocyte function in vitro. Reduction of lysosomal enzyme release and superoxide anion production. $J$ Exp Med 146, 483-494.

Himes, R.S., Jr., DiFazio, C.A. and Burney, R.G. (1977) Effects of lidocaine on the anesthetic requirements for nitrous oxide and halothane. Anesthesiology 47, 437440 .

Kalff, J., Schraut, W. and Simmons, R. (1998) Surgical manipulation of the gut elicits an intestinal muscularis inflammatory response resulting in post surgical ileus. Ann Surg 228, 652-663.

Koenig, J. and Cote, N. (2006) Equine gastrointestinal motility--ileus and pharmacological modification. Can Vet J 47, 551-559.

Lauretti, G.R. (2008) Mechanisms of analgesia of intravenous lidocaine. Rev Bras Anestesiol 58, 280-286.

Lei, B., Cottrell, J.E. and Kass, I.S. (2001) Neuroprotective effect of low-dose lidocaine in a rat model of transient focal cerebral ischemia. Anesthesiology 95, 445-451.

LeLorier, J., Grenon, D., Latour, Y., Caille, G., Dumont, G., Brosseau, A. and Solignac, A. (1977a) Pharmacokinetics of lidocaine after prolonged intravenous infusions in uncomplicated myocardial infarction. Ann Intern Med 87, 700-706.

LeLorier, J., Moisan, R., Gagne, J. and Caille, G. (1977b) Effect of the duration of infusion on the disposition of lidocaine in dogs. J Pharmacol Exp Ther 203, 507511.

Liu, K., Adachi, N., Yanase, H., Kataoka, K. and Arai, T. (1997) Lidocaine suppresses the anoxic depolarization and reduces the increase in the intracellular $\mathrm{Ca} 2+$ concentration in gerbil hippocampal neurons. Anesthesiology 87, 1470-1478.

Malone, E., Ensink, J., Turner, T., Wilson, J., Andrews, F., Keegan, K. and Lumsden, J. (2006) Intravenous continuous infusion of lidocaine for treatment of equine ileus. Vet Surg 35, 60-66.

Malone, E. and Graham, L. (2002) Management of gastrointestinal pain. Vet Clin North Am Equine Pract 18, 133-158.

Malone, E., Turner, T. and Wilson, J. (1998) Intravenous lidocaine for the treatment of equine ileus. In: 6th Equine Colic Research Symposium, Athens, GA. p 42. 
Mama, K. and Steffey, E. (2001) Drugs acting in the central nervous system, Local anesthetics. In: Veterinary Pharmacology and therapeutics, 8th edn., Ed: A. HR, Iowa State University Press, Ames, Iowa. pp 339-355.

Mather, L.E., Runciman, W.B., Carapetis, R.J., Ilsley, A.H. and Upton, R.N. (1986) Hepatic and renal clearances of lidocaine in conscious and anesthetized sheep. Anesth Analg 65, 943-949.

McKindley, D.S., Boulet, J., Sachdeva, K., Wang, P. and Chichester, C. (2002) Endotoxic shock alters the pharmacokinetics of lidocaine and monoethylglycinexylidide. Shock 17, 199-204.

Meyer, G.A., Lin, H.C., Hanson, R.R. and Hayes, T.L. (2001) Effects of intravenous lidocaine overdose on cardiac electrical activity and blood pressure in the horse. Equine Vet J 33, 434-437.

Milligan, M., Beard, W., Kukanich, B., Sobering, T. and Waxman, S. (2007) The effect of lidocaine on postoperative jejunal motility in normal horses. Vet Surg 36, 214220.

Milligan, M., Kukanich, B., Beard, W. and Waxman, S. (2006) The disposition of lidocaine during a 12-hour intravenous infusion to postoperative horses. $J$ Vet Pharmacol Ther 29, 495-499.

Muir, W. and McGuirk, S. (1985) Pharmacology and pharmacokinetics of drugs used to treat cardiac disease in horses. The Veterinary Clinics of North America Equine Practice 1, 335-352.

Murrell, J.C., White, K.L., Johnson, C.B., Taylor, P.M., Doherty, T.J. and WatermanPearson, A.E. (2005) Investigation of the EEG effects of intravenous lidocaine during halothane anaesthesia in ponies. Vet Anaesth Analg 32, 212-221.

Nadrowski, L. (1983) Paralytic ileus: recent advances in pathophysiology and treatment. Current Surgery 40, 260-273.

Nieto, J.E., Rakestraw, P.C., Snyder, J.R. and Vatistas, N.J. (2000) In vitro effects of erythromycin, lidocaine, and metoclopramide on smooth muscle from the pyloric antrum, proximal portion of the duodenum, and middle portion of the jejunum of horses. Am J Vet Res 61, 413-419.

Oellerich, M. and Armstrong, V.W. (2001) The MEGX test: a tool for the real-time assessment of hepatic function. Ther Drug Monit 23, 81-92.

Oellerich, M., Raude, E., Burdelski, M., Schulz, M., Schmidt, F.W., Ringe, B., Lamesch, P., Pichlmayr, R., Raith, H., Scheruhn, M., Wrenger, M. and Wittekind, C. (1987) 
Monoethylglycinexylidide formation kinetics: a novel approach to assessment of liver function. J Clin Chem Clin Biochem 25, 845-853.

Pfeifer, H.J., Greenblatt, D.J. and Koch-Weser, J. (1976) Clinical use and toxicity of intravenous lidocaine. A report from the Boston Collaborative Drug Surveillance Program. Am Heart J 92, 168-173.

Pieper, J.A., Slaughter, R.L., Anderson, G.D., Wyman, M.G. and Lalka, D. (1982) Lidocaine clinical pharmacokinetics. Drug Intell Clin Pharm 16, 291-294.

Rimback, G., Cassuto, J. and Tollesson, P.O. (1990) Treatment of postoperative paralytic ileus by intravenous lidocaine infusion. Anesth Analg 70, 414-419.

Robertson, S.A. (2004) Standing sedation and pain management for ophthalmic patients. Vet Clin North Am Equine Pract 20, 485-497.

Robertson, S.A., Sanchez, L.C., Merritt, A.M. and Doherty, T.J. (2005) Effect of systemic lidocaine on visceral and somatic nociception in conscious horses. Equine Vet J 37, 122-127.

Roussel, A.J., Jr., Cohen, N.D., Hooper, R.N. and Rakestraw, P.C. (2001) Risk factors associated with development of postoperative ileus in horses. J Am Vet Med Assoc 219, $72-78$.

Rusiecki, K.E., Nieto, J.E., Puchalski, S.M. and Snyder, J.R. (2008) Evaluation of continuous infusion of lidocaine on gastrointestinal tract function in normal horses. Vet Surg 37, 564-570.

Sasyniuk, B.I. and Ogilvie, R.I. (1975) Antiarrhythmic drugs: electrophysiological and pharmacokinetic considerations. Annu Rev Pharmacol 15, 131-155.

Scott, S.M., Knowles, C.H., Wang, D., Yazaki, E., Picon, L., Wingate, D.L. and Lindberg, G. (2006) The nocturnal jejunal migrating motor complex: defining normal ranges by study of 51 healthy adult volunteers and meta-analysis. Neurogastroenterol Motil 18, 927-935.

Siddall, P.J. and Cousins, M.J. (1995) Pain mechanisms and management: an update. Clin Exp Pharmacol Physiol 22, 679-688.

Smith, E.R. and Duce, B.R. (1971) The acute antiarrhythmic and toxic effects in mice and dogs of 2-ethylamino-2',6-'acetoxylidine (L-86), a metabolite of lidocaine. $J$ Pharmacol Exp Ther 179, 580-585.

Sotgiu, M.L., Lacerenza, M. and Marchettini, P. (1992) Effect of systemic lidocaine on dorsal horn neuron hyperactivity following chronic peripheral nerve injury in rats. Somatosens Mot Res 9, 227-233. 
Stenson, R.E., Constantino, R.T. and Harrison, D.C. (1971) Interrelationships of hepatic blood flow, cardiac output, and blood levels of lidocaine in man. Circulation 43, 205-211.

Tam, Y.K., Yau, M., Berzins, R., Montgomery, P.R. and Gray, M. (1987) Mechanisms of lidocaine kinetics in the isolated perfused rat liver. I. Effects of continuous infusion. Drug Metab Dispos 15, 12-16.

Taniguchi, T., Shibata, K., Yamamoto, K., Kobayashi, T., Saito, K. and Nakanuma, Y. (1996) Lidocaine attenuates the hypotensive and inflammatory responses to endotoxemia in rabbits. Crit Care Med 24, 642-646.

Taylor, C.P., Burke, S.P. and Weber, M.L. (1995) Hippocampal slices: glutamate overflow and cellular damage from ischemia are reduced by sodium-channel blockade. J Neurosci Methods 59, 121-128.

Terada, H., Ohta, S., Nishikawa, T., Mizunuma, T., Iwasaki, Y. and Masaki, Y. (1999) The effect of intravenous or subarachnoid lidocaine on glutamate accumulation during transient forebrain ischemia in rats. Anesth Analg 89, 957-961.

Thomasy, S.M., Pypendop, B.H., Ilkiw, J.E. and Stanley, S.D. (2005) Pharmacokinetics of lidocaine and its active metabolite, monoethylglycinexylidide, after intravenous administration of lidocaine to awake and isoflurane-anesthetized cats. Am J Vet Res 66, 1162-1166.

Thomson, A.H., Elliott, H.L., Kelman, A.W., Meredith, P.A. and Whiting, B. (1987) The pharmacokinetics and pharmacodynamics of lignocaine and MEGX in healthy subjects. J Pharmacokinet Biopharm 15, 101-115.

Thomson, P.D., Melmon, K.L., Richardson, J.A., Cohn, K., Steinbrunn, W., Cudihee, R. and Rowland, M. (1973) Lidocaine pharmacokinetics in advanced heart failure, liver disease, and renal failure in humans. Ann Intern Med 78, 499-508.

Tremont-Lukats, I.W., Hutson, P.R. and Backonja, M.M. (2006) A randomized, doublemasked, placebo-controlled pilot trial of extended IV lidocaine infusion for relief of ongoing neuropathic pain. Clin J Pain 22, 266-271.

Tucker, G.T., Boyes, R.N., Bridenbaugh, P.O. and Moore, D.C. (1970) Binding of anilide-type local anesthetics in human plasma. II. Implications in vivo, with special reference to transplacental distribution. Anesthesiology 33, 304-314.

Valverde, A., Gunkelt, C., Doherty, T.J., Giguere, S. and Pollak, A.S. (2005) Effect of a constant rate infusion of lidocaine on the quality of recovery from sevoflurane or isoflurane general anaesthesia in horses. Equine Vet J 37, 559-564. 
Waller, E.S. (1981) Pharmacokinetic principles of lidocaine dosing in relation to disease state. J Clin Pharmacol 21, 181-194.

Weiker, G.G., Kuivila, T.E. and Pippinger, C.E. (1991) Serum lidocaine and bupivacaine levels in local technique knee arthroscopy. Am J Sports Med 19, 499-502.

\footnotetext{
${ }^{\mathrm{i}}$ Equimax (Ivermectin/praziquantel), Virbac AH Inc Fort Worth, TX USA

${ }^{i i}$ Lidocaine 20\%, VEDCO Inc., St Joseph MO USA

iii Abbott Laboratories, North Chicago IL USA

${ }^{\text {iv }}$ IMED PC-2, IMED Corporation, San Diego, CA, USA

${ }^{v}$ International Win Ltd, Kennett Square PA, USA

${ }^{\text {vi }}$ Milacath, Mila International Inc, Erlanger, KY, USA

${ }^{v i i}$ Carbocaine, Pfizer Inc, NY, USA

viii Becton Dickson, Franklin Lakes NJ, USA

${ }^{i x}$ University of Tennessee College of Veterinary Medicine, Department of Comparative Medicine, Knoxville, TN, USA

${ }^{\mathrm{x}}$ Waters Corp., Milford, MA, USA
} 